\title{
Limits on the early afterglow phase of gamma-ray burst sources from TAROT-1
}

\author{
M. Boër ${ }^{1}$, J. L. Atteia ${ }^{1}$, M. Bringer ${ }^{1}$, B. Gendre ${ }^{1}$, A. Klotz ${ }^{1}$, R. Malina ${ }^{2}$, \\ J. A. de Freitas Pacheco ${ }^{3}$, and H. Pedersen ${ }^{4}$ \\ 1 Centre d'Étude Spatiale des Rayonnements (CNRS/UPS), BP 4346, 31028 Toulouse Cedex 4, France \\ 2 Laboratoire d'Astrophysique de Marseille (CNRS/OAMP), Traverse du Siphon, BP 8, \\ 3 Observatoire de la Côte d'Azur, BP 229, 06304 Nice Cedex 4, France \\ 4 NBIfAFG, Copenhagen University Observatory, Juliane Maries Vej 30, 2100 Copenhagen Ø, Denmark
} 13376 Marseille Cedex 12, France

Received 30 October 2000 / Accepted 9 August 2001

\begin{abstract}
The Télescope à Action Rapide pour les Objets Transitoires (TAROT-1) has as its prime objective the observation of the prompt and delayed emission of cosmic gamma-ray bursts (GRBs). We have performed a search for optical emission from 6 GRBs detected by BATSE. The positioning error circle was fully covered within typically thirty minutes after the trigger. No detection of the early afterglow phase was made, and magnitude limits in the range of $m_{R}=13-15$ were estimated using $20 \mathrm{~s}$ exposures. These limits are compared to optical afterglow data obtained in later phases and the results are interpreted in terms of source distances. They correspond to a median redshift of $z=0.5$. With HETE- 2 and the planned instrument upgrade, TAROT- 1 will be able to detect the early optical emission of GRBs up to a redshift of the order of 5 .
\end{abstract}

Key words. gamma-ray: bursts

\section{Introduction}

Since their discovery more than thirty years ago, GammaRay Bursts (GRBs) have been intriguing objects for theoreticians and challenging sources for observers. Their cosmological nature was firmly established in 1997 by observations performed by the BeppoSAX satellite (Costa 1999), which enabled the first discovery of a faint optical transient associated with the event (van Paradijs et al. 1997). With the possible exception of GRB 980425, whose association with SN 1998bw remains in doubt (Pian et al. 1999), the measured GRB source redshifts are close to, or larger than, unity. The events may be decomposed, in general, in two main phases: a prompt bursting phase, with no preferred temporal pattern, with a duration ranging from few milliseconds to several minutes (Paciesas et al. 1999); a delayed emission, the afterglow, that to a first approximation decays according to a power-law of index $\approx-1$. BeppoSax wide-field observations were of fundamental importance for the discovery and the follow-up of the afterglow phase at different wavelengths (Costa et al. 1997; van Paradijs et al. 1997). The ROTSE experiment has provided evidence for emission at optical frequencies from the prompt phase, detecting for the first time an optical burst occurring during the gamma-ray emission of GRB 990123 (Akerlof et al. 2000).

Send offprint requests to: M. Boër, e-mail: Michel.Boer@cesr.fr
The detection of X-ray and optical afterglows of cosmic gamma-ray bursts supports the fireball model (Rees \& Mészáros 1992; Mészáros \& Rees 1997; Panaitescu et al. 1998) as a standard tool to interpret those observations. In this framework, the afterglow emission is described as synchrotron and inverse Compton emission of high energy electrons accelerated during the shock of an ultrarelativistic shell with the external medium, while the prompt emission is due to the internal shocks produced by shells at different speeds within the relativistic blast wave (see Piran 1999 for a review). Both the prompt radiation and early afterglow phases provide us with critical information to establish the physical processes during the burst itself, as well as the physical conditions of the surrounding environment (Kumar \& Panaitescu 2000; Kumar \& Piran 2000). There is general consensus that the fireball plasma is constituted of $\mathrm{e}^{-} \mathrm{e}^{+}$pairs and $\gamma$-photons, however the ultimate energy reservoir and the mechanism of pair creation are still a challenge to theoretical models.

Since 1991 the Burst and Transient Experiment (BATSE, Fishman 1989) on board the Compton GammaRay Observatory (CGRO) has been detecting about one GRB source per day in the 50-300 keV energy band, with fluences ranging from $10^{-7} \mathrm{erg} \mathrm{cm}^{-2}$ to $10^{-3} \mathrm{erg} \mathrm{cm}^{-2}$. The GRB Coordinate Network (GCN, Barthelmy 1997) uses the raw real-time data of BATSE to compute very rapidly an approximate position of the sources. In spite of the large error circle of the initial coordinates, $5^{\circ}$ or more, 
Table 1. Main technical characteristics of TAROT-1.

\begin{tabular}{ll}
\hline Aperture & $25 \mathrm{~cm}$ \\
Field of view & $1^{\circ} \times 1^{\circ}$ \\
Optical resolution & $20 \mu \mathrm{m}$ \\
Mount type & equatorial \\
Axis speed $(\alpha$ and $\delta)$ & $\begin{array}{l}\text { adjustable, up to } 80^{\circ} / \mathrm{s} \\
\text { CCD type }\end{array}$ \\
Apogee $A M 13$ with Kodak $K A F 1300$ \\
Pixel size & $1080 \times 1280$ pixels \\
CCD readout noise & $15 \mu \mathrm{m}$ \\
Readout time & $300 \mathrm{~s}$ \\
Filter wheel & 6 pos. : Clear, $V, R, I, B+V, R+I^{\mathrm{a}}$ \\
\hline
\end{tabular}

${ }^{\text {a }}$ Filters $B+V$ and $R+I$ are broad band filters covering the spectral range of respectively the Cousin $B$ and $V$, and $R$ and $I$ filters.

the information on the source position is transmitted in a very short time, about $4 \mathrm{~s}$ after the burst triggering by the BATSE detectors. Wide field telescopes equipped with rapid optical detectors may quickly point and monitor the source position, searching for an optical transient, like the detection of GRB 990123 by the ROTSE experiment (Akerlof et al. 1999).

The aim of the Télescope à Action Rapide pour les Objets Transitoires (Rapid Action Telescope for Transient Objects, hereafter TAROT-1, Boër et al. 1999) is the rapid response to a triggered $\gamma$-ray event, searching for the detection of the prompt optical counterpart as well as of the early phases of the afterglow. Although this experiment was primarily designed to work in conjunction with the HETE satellite (Ricker et al. 2001), its wide field permits the observation of BATSE/GCN alerts using multiple pointings. In this paper we present the limits obtained for the early afterglow phases of sources observed during the period 1999-2000 detected by BATSE. These limits are interpreted in light of data obtained on GRB afterglows and in the framework of the fireball model.

\section{The instrument}

TAROT-1 is a fully autonomous $25 \mathrm{~cm}$ aperture telescope. Its $2^{\circ}$ field of view matches well the HETE uncertainty in localization of the sources (particularly for the HETE-1 WXM, since TAROT-1 was designed before its unsuccessful launch). Table 1 summarizes the present main technical characteristics of TAROT-1.

TAROT-1 receives about one observable alert from BATSE every ten days. This is in agreement with the BATSE statistics of GRB event detection, the relative night-day duration and the accessible area of the sky $\left(10^{\circ}\right.$ above the North horizon to $20^{\circ}$ for the South part of the sky). Weather conditions prevent observation during $30 \%$ of the time. Observations are performed even during the full Moon periods, although with a reduced sensitivity. TAROT-1 is fully autonomous i.e. there is practically no human intervention. Figure 1 summarizes the different software modules and their connections. All are function-

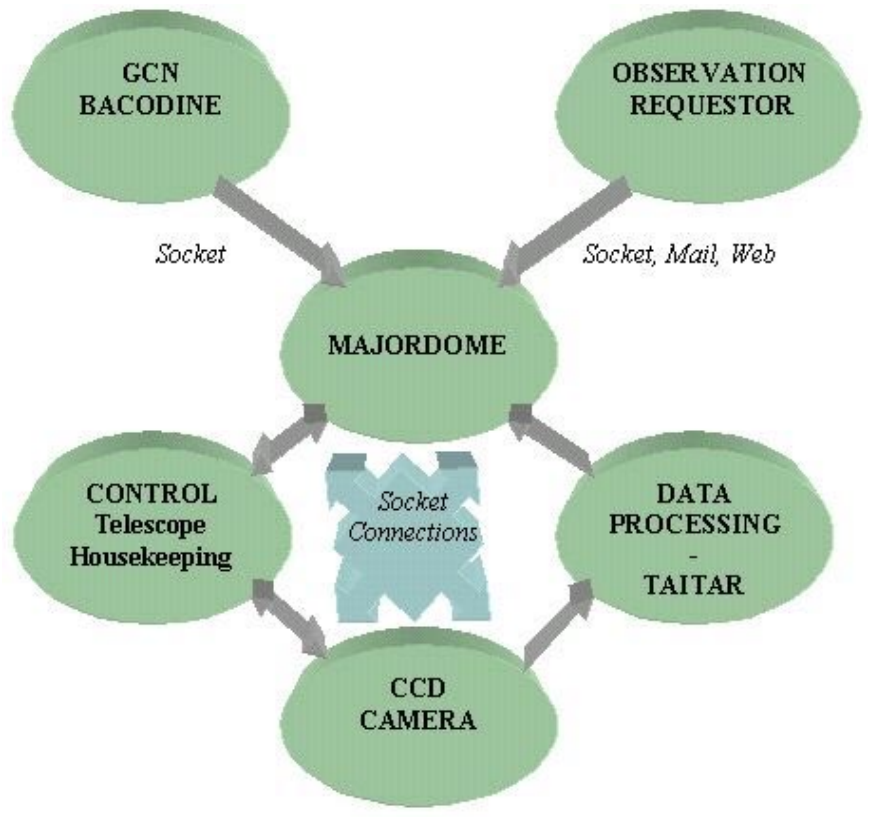

Fig. 1. The software modules of TAROT-1 and their interactions from Bringer et al. (2001). In the case of GRBs, requests are sent to the MAJORDOME by the GCN (BACODINE). The observations are immediately scheduled, and processed on line by the TAITAR software.

ally independent and the communication is made through TCP/IP socket processes.

The Telescope Control System (TCS) takes care of the instrument, performs housekeeping, weather monitoring, etc. During the night, the control is handed over by the MAJORDOME (Bringer et al. 2001), a software package in charge of the observations. In the absence of any alert from the GCN, the instrument is in routine mode, and observations requested by various observers are sequenced. Upon receipt of an observable GCN alert from BATSE, the ongoing observation is interrupted, and the MAJORDOME sends the telescope and camera parameters to the TCS. The reconfiguration and pointing of the telescope to its new position lasts typically $1.5 \mathrm{~s}$. Taking into account the various delays involved by the GCN (4-6 s), and the INTERNET socket transmission (typically $0.25 \mathrm{~s}$ ), the total reaction time, from the time of the BATSE trigger to the beginning of data acquisition, is about of $7-10 \mathrm{~s}$. Since the positioning error circle of BATSE is larger than the telescope field of view, observations are performed inside a field of $25^{\square}$, covered by a set of $5 \times 5$ mosaic. In the best cases this total field covers only the $68 \%$ confidence radius of the error circle, including statistical errors, as discussed in the BATSE 4B catalog (Paciesas et al. 1999). TAROT-1 reacts to BATSE_LOCBURST notices (see the GCN web site: http://gcn.gsfc.nasa.gov for a description of the various notice types). After usually $20 \mathrm{~s}$ to $10 \mathrm{~min}$, a new alert is sent by the GCN, called BATSE_FINAL, with improved coordinates, producing a second positioning of TAROT-1, and the start of a new mosaic. If the difference between the BATSE_LOCBURST and BATSE_FINAL positions is less 
Table 2. TAROT-1 observation log for the GRB source observations made with CGRO-BATSE.

\begin{tabular}{|c|c|c|c|c|c|}
\hline Source name & $\begin{array}{l}\text { BATSE trigger } \\
\text { time (UT) }\end{array}$ & $\begin{array}{l}\text { Error circle } \\
\operatorname{radius}\left({ }^{\circ}\right)^{\mathrm{a}}\end{array}$ & $\begin{array}{l}\text { Matching with mosaic? } \\
\text { (see text) }\end{array}$ & Sky conditions & $\begin{array}{l}\text { Moon phase } \\
(\%)\end{array}$ \\
\hline GRB 990802 & 01:04:44 & 2.1 & Full & Clear & 70 \\
\hline GRB 990807 & $21: 55: 46$ & 2.5 & No match & Clear & No Moon \\
\hline GRB 990903 & $19: 14: 56$ & 2.7 & Partial & Partly cloudy & No Moon \\
\hline GRB 990915 & $23: 15: 23$ & 2.2 & Full & Clear & No Moon \\
\hline GRB 990917 & $20: 12: 28$ & 2.6 & Partial & Clear & 50 \\
\hline GRB 991002 & $22: 49: 03$ & 2.8 & Full & Clear & 45 \\
\hline GRB 991115 & $18: 48: 08$ & 2.2 & Partial & Cloudy & 45 \\
\hline GRB 991129 & $22: 38: 08$ & 4.0 & No match & Clear & 50 \\
\hline GRB 991211 & $04: 34: 42$ & 6.1 & No match & Clear & No Moon \\
\hline GRB 000107 & 00:44:09 & 6.8 & No match & Clear & No Moon \\
\hline GRB 000111 & $02: 41: 58$ & 5.2 & Partial & Clear & No Moon \\
\hline GRB 000201 & 03:02:09 & 2.1 & Full & Clear & 25 \\
\hline GRB 000221 & $23: 39: 10$ & 2.0 & No match & Partly cloudy & 85 \\
\hline GRB 000225 & $01: 35: 29$ & 2.3 & Full & Partly cloudy & 62 \\
\hline GRB 000226 & $03: 51: 49$ & 2.5 & Partial & Clear & 55 \\
\hline GRB 000229 & $02: 45: 02$ & 2.4 & Full & Cirrus clouds? & 35 \\
\hline GRB 000302 & $02: 50: 25$ & 2.1 & No match & Clear & No Moon \\
\hline GRB 000312 & $20: 50: 50$ & 2.5 & Full & Clear & 45 \\
\hline GRB 000321 & 21:53:08 & 4.1 & Partial & Partly cloudy & 90 \\
\hline GRB 000408 & $02: 35: 48$ & 2.5 & Partial & Clear & No Moon \\
\hline GRB 000418 & $21: 30: 17$ & 5.4 & No match & Clear & 100 \\
\hline
\end{tabular}

a $68 \%$ confidence radius from the last available BATSE data, taking into account both statistical and systematic errors.

than $1^{\circ}$ the telescope keeps its current position. Depending on the burst brightness, the Huntsville BATSE team may compute a better position, which is usually transmitted by the GCN 30 to 60 min later. TAROT-1 moves accordingly and eventually reduces the area explored, depending on the actual uncertainty. During the remainder of the night, a continuous coverage of the area is maintained, trying to detect and to keep track of an eventual optical transient.

As soon as an image is obtained, the image processing software (called TAITAR in Fig. 1) produces a source catalog. Presently, the generated list of sources is compared to the USNO catalog both for astrometric and photometric reductions. Once TAROT-1 has completed a full mosaic, an e-mail message is sent, and a scientist on duty is paged to compare the images and to look for possible new sources. The images are acquired without filter (clear position in the filter wheel) and with an exposure time of $20 \mathrm{~s}$. With the APOGEE camera the readout time was $30 \mathrm{~s}$, hence the total time needed to observe the full mosaic was $21 \mathrm{~min}$.

\section{Observations and reduction}

TAROT-1 observations of GRBs started routinely in 1999, although the data for the first half of July 1999 has been lost because of a hardware failure of the media recorder. All observations are summarized in Table 2. The two first columns give the GRB date and time. The third column gives the error circle taken from the final data reduction provided by the BATSE team, including the systematic error as given in Paciesas et al. (1999). For several sources, it appears that neither the BATSE_FINAL nor the BATSE_LOCBURST positions were accurate enough to match fully our mosaic. This indication is given in Col. 4, while the sky conditions are depicted in the next column, with the Moon phase in the 6th column. The influence of the Moon depends on its phase and distance to the source, and has an impact on the upper limits we computed. A phase of $30 \%$ begin to produce a higher background, whatever the Moon position on the sky. No event observed by TAROT-1 as a response to a BATSE/GCN alert was detected by another instrument, whatever the wavelength. In six cases, the $68 \%$ error circle had been adequately sampled and the sky conditions were correct, hence the probability that TAROT-1 never observed a burst source (i.e. the six sources are outside their $68 \%$ error circle) is 0.0011 , assuming a uniform probability distribution over the error circle. Of course, the detection probability depends on the unknown source magnitude. Hence the source detection probability will be $(1-0.68 p)^{6}$, where $p$ represents the fraction of bursts above the TAROT- 1 detection threshold at the time of observation.

Since no optical sources were detected, a $3 \sigma$ upper limit to the magnitude was calculated by comparing several stars on the images with the USNO A2.0 catalog. Since observations were performed without any filter, a correction factor was estimated in order to provide a $R$-magnitude upper limit, assuming an energy spectral index equal to 1. Under this condition, one finds that the correction to be applied to the measured TAROT magnitude $m_{\mathrm{T}}$ is $R=m_{\mathrm{T}}-0.4$. The limits obtained are listed in Table 3 as 
Table 3. $3 \sigma$ upper limits (transformed in $R$ magnitude) to the GRB source counterparts for the 6 well-observed events mentioned in Table 2.

\begin{tabular}{lll}
\hline Source name & $\begin{array}{l}\text { Time since trigger } \\
(\text { days })\end{array}$ & $\begin{array}{l}3 \sigma \text { upper limit } \\
(R \text { magnitude })\end{array}$ \\
\hline GRB 990802 & 0.0200 & 12.7 \\
& 0.0402 & 13.2 \\
GRB 990915 & 0.0404 & 13.9 \\
& 0.0719 & 14.1 \\
GRB 991002 & 0.0231 & 13.0 \\
& 0.0441 & 12.9 \\
& 0.0643 & 14.3 \\
& 0.0971 & 14.2 \\
GRB 000201 & 0.1299 & 12.8 \\
& 0.0177 & 13.3 \\
& 0.0317 & 15.3 \\
& 0.0504 & 15.3 \\
GRB 000229 & 0.0723 & 15.1 \\
& 0.0885 & 14.9 \\
GRB 000312 & 0.0192 & 14.2 \\
\hline
\end{tabular}

a function of the elapsed time since the BATSE trigger. The delays mentioned in the second column corresponds to the end of the scan matching the final BATSE source position.

\section{Discussion}

TAROT-1 upper limits on the optical flux of GBR 990123, the only source until now detected both during its prompt and afterglow phase, were compared with ROTSE data. Figure 2 displays TAROT- 1 upper limits and ROTSE measurements (Akerlof et al. 1999), as well as later data (Galama et al. 1999).

Another possibility is to compare TAROT-1 upper limits with data obtained for GRB afterglows at later times. The result of this comparison is displayed in Fig. 3, whereas the basic data and references are given in Table 4. We note that in the time frame observed by TAROT-1, GRB 970508, GRB 980519, GRB 991208, and GRB 991216, in addition to GRB 990123, would have been detected by TAROT, if we extrapolate the late epoch afterglow to early times. Of course, this is a crude extrapolation which does not take into account possible breaks in the light curves.

We tried to evaluate the possible effect of the source distance on the burst afterglow detection by TAROT-1. To perform this task we have to correct for the galactic absorption and for the source distance (Boër \& Gendre 2000). We have scaled the flux of sources with known distances to a common redshift of $z=1$, applying corrections for distance, time dilation and absorption in our galaxy (Schlegel et al. 1998). We assume a flat cosmology with $\Omega_{\Lambda}=0.7$ and $H_{0}=65 \mathrm{~km} \mathrm{~s}^{-1} \mathrm{Mpc}^{-1}$. Table 5 gives
Table 4. The GRB source sample used for comparison of the known afterglow light curves with our upper limits. Column 3 indicates the decay index after correction.

\begin{tabular}{lll}
\hline Source & Redshift & References \\
\hline GRB 970228 & 0.695 & $1,2,3,4$ \\
GRB 970508 & 0.835 & $5-10$ \\
GRB 971214 & 3.42 & 11 \\
GRB 980329 & - & 12 \\
GRB 980519 & - & $13-15$ \\
GRB 980613 & 1.096 & $16-18$ \\
GRB 980703 & 0.966 & 19 \\
GRB 990123 & 1.61 & 20,21 \\
GRB 990510 & 1.62 & 22,23 \\
GRB 991208 & - & $24-26$ \\
GRB 991216 & 1.02 & $27-33$ \\
GRB 000301c & - & $34-40$ \\
GRB 000418 & 1.12 & $41-43$ \\
\hline
\end{tabular}

1. Guarnieri et al. (1997), 2. Metzger et al. (1997), 3. Pedichini et al. (1997), 4. van Paradijs et al. (1997), 5. Fruchter et al. (1997), 6. Galama et al. (1997), 7. Garcia et al. (1997), 8. Djorgovsky et al. (1997), 9. Mignoli et al. (1997), 10. Pedersen et al. (1998), 11. Diercks et al. (1998), 12. Reichart et al. (1999), 13. Bloom et al. (1998a), 14. Gal et al. (1998), 15. Vrba et al. (2000), 16. Djorgovsky et al. (1998), 17. Halpern et al. (1998), 18. Hjorth et al. (1998), 19. Bloom et al. (1998b), 20. Akerlof et al. (1999), 21. Galama et al. (1999), 22. Harrison et al. (1999), 23. Staneck et al. (1999), 24. Kuulkers et al. (2000), 24. Castro-Tirado et al. (1999), 25. Jensen et al. (1999a), 26. Masetti et al. (1999), 27. Djorgovsky et al. (1999), 28. Dolan et al. (1999), 29. Garnavich et al. (1999), 30. Henden et al. (1999), 31. Jensen et al. (1999b), 32. Jha et al. (1999), 33. Sagar et al. (2000), 34. Bernabei et al. (2000), 35. Fynbo et al. (2000), 36. Gal-Yam et al. (2000), 37. Garnavich et al. (2000), 38. Halpern et al. (2000), 39. Mohan et al. (2000), 40. Veillet \& Boër (2000), 41. Henden (2000), 42. Henden \& Klose (2000), 43. Mirabal et al. (2000).

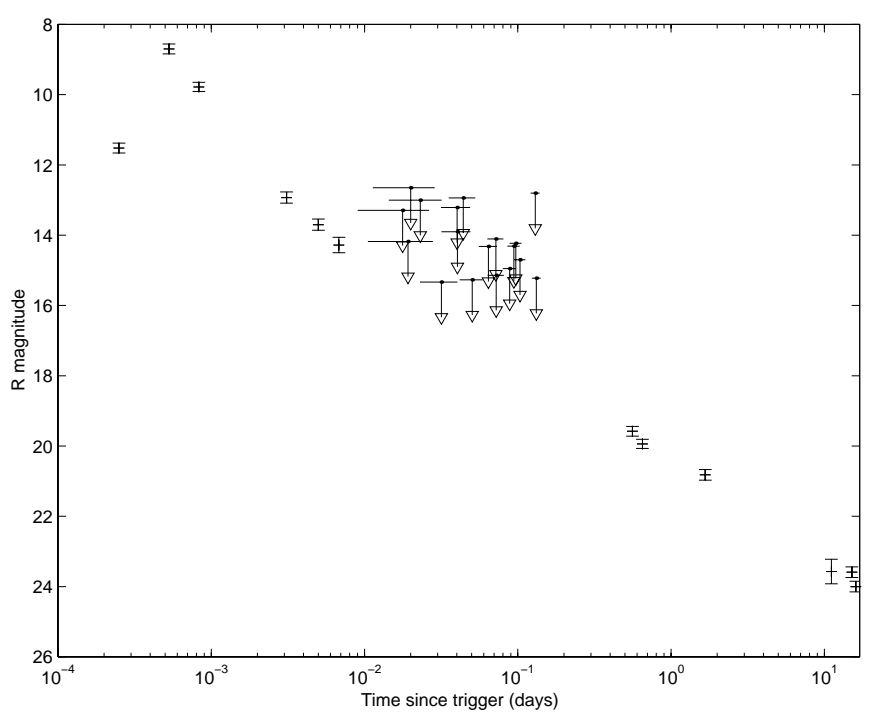

Fig. 2. Comparison between the TAROT upper limits (arrows) and the light curve of GRB 990123 (error bars). Both the prompt and the afterglow emission are shown. Data from Akerlof et al. (1999) and from Galama et al. (1999). 
Table 5. Distance and galactic absorption correction factors for the GRB sources of known distance. These quantities are used to normalize the GRB light curves to a common distance corresponding to a redshift of 1 (see text). The corrected power law decay index is tabulated Col. 5 (for details see Boër \& Gendre 2000).

\begin{tabular}{lllll}
\hline \multicolumn{4}{c}{ Correction factors } \\
Source & $\begin{array}{l}\text { Correction for } \\
\text { distance }\end{array}$ & $K$-Correction & $\begin{array}{l}\text { Galactic } \\
\text { absorption }\end{array}$ & $\begin{array}{l}\text { Corrected light curve } \\
\text { decay index }\end{array}$ \\
\hline GRB 970228 & 0.41 & 1.0 & 0.61 & $1.10 \pm 0.11$ \\
GRB 970508 & 0.64 & 1.0 & 0.05 & $1.17 \pm 0.21$ \\
GRB 971214 & 20.2 & 1.2 & 0.06 & $1.20 \pm 0.27$ \\
GRB 980613 & 1.10 & 1.0 & 0.22 & $1.00 \pm 0.01$ \\
GRB 980703 & 0.92 & 1.0 & 0.72 & $1.17 \pm 0.25$ \\
GRB 990123 & 3.23 & 0.9 & 0.04 & $1.44 \pm 0.07$ \\
GRB 990510 & 3.28 & 0.9 & 0.53 & $1.54 \pm 0.15$ \\
GRB 991216 & 1.05 & 1.0 & 1.67 & $1.22 \pm 0.04$ \\
\hline
\end{tabular}

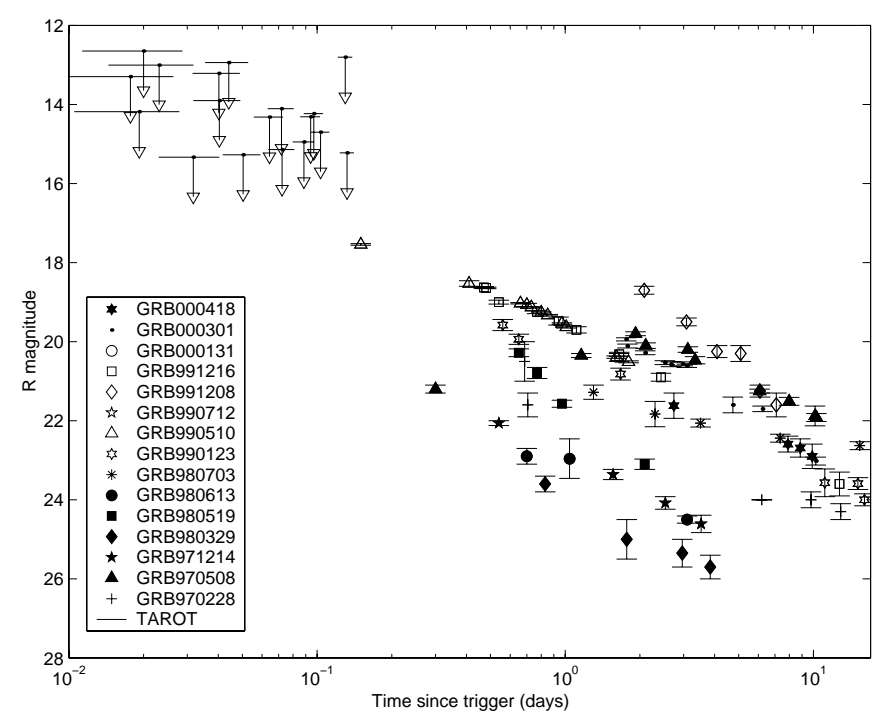

Fig. 3. Comparison between the TAROT upper limits (arrows, all data) and the afterglow light curve of GRB sources detected at optical wavelengths (Table 4). In this figure, the light curves have not been corrected.

the corrections computed and the decay indexes. The corrected light curves are displayed in Fig. 4 together with TAROT-1 upper limits. As it can be seen, extrapolating to the proper time domain, TAROT-1 would have detected 5 events, if their redshift would have been equal to $z=1$.

These data can also be used to estimate the limiting TAROT-1 distance of detection. Assuming a limiting magnitude of 15 one hour after the burst, the corresponding distance is $z=1.2$ for the brightest burst and 0.1 for the faintest one, the median redshift being 0.5 .

\section{Conclusions and perspectives}

In this paper, the lower $R$-magnitude limit for the early afterglow light curves of GRBs is computed, for sources observed by TAROT-1 after its first year of routine operation. These limits are compared with the optical afterglow data obtained so far. A maximum source distance of $z=1.2$ was estimated for observations made with

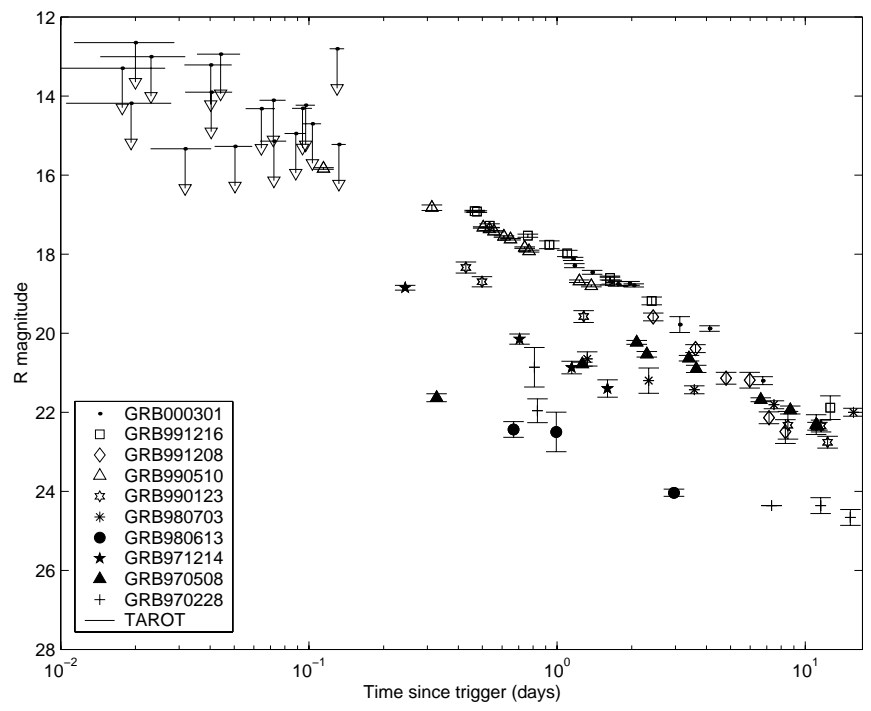

Fig. 4. Comparison between the TAROT upper limits (arrows, all data) and the afterglow light curve of GRB sources. The light curves have been normalized to a common distance corresponding to a redshift of 1 , according to the method described in the text (Boër \& Gendre 2000) and using the data from Table 5 . We took into account the $K$-correction, distance and time dilation, and absorption from our Galaxy (Table 5).

TAROT-1 one hour after the GRB main event begins. The accuracy of HETE-2 (Ricker et al. 2001) or SWIFT (Gehrels 2000) will allow to start the observation within few seconds after the GRB triggers the detectors, while for BATSE we had to scan the error circle, an operation lasting $30 \mathrm{~min}$. Given the expected HETE-2 detection rate of 40 events per year in the anti-solar direction, TAROT-1 may expect to observe a source location once every month. In order to estimate the maximum source distance TAROT-1 can reach, we extrapolate the light curves plotted in Fig. 4 to a time of five minutes after the burst onset. In this case, the median redshift corresponding to a limiting magnitude of 15 is $z=1.2$.

In addition, the detector will soon be replaced by a camera based on a Thomson 7899 CCD. It will have a total noise of $10 \mathrm{e}^{-}$, for a readout time of $1 \mathrm{~s}$, allowing it to 
reach the 17 th $R$ magnitude in $10 \mathrm{~s}$. With this new device TAROT-1 will be able to monitor the GRB optical light curves, not only during the prompt event, but also the transition between the prompt and afterglow phase. After five minutes, even in the worst case discussed in Sect. 4 ( $z=0.1$, one hour after the burst onset with the previous camera) the TAROT-1 distance limit will correspond to a redshift of 2 , and the median redshift of 5.6, while after 1 hour, this distance will still correspond to a median redshift of 2.2 (0.5 with the previous camera).

Already, in its current configuration TAROT-1 is able to give stringent limits to the early afterglow phase. In conjunction with a satellite like HETE-2 or SWIFT, the transition between the prompt and delayed mode will be monitored, allowing us to acquire critical data on GRB sources.

Acknowledgements. We would like to pay tribute to our collaborator Gérard Calvet, engineer at the INSU/DT, who tragically died during a mission at the Plateau de Bure IRAM observatory. The Télescope à Action Rapide pour les Objets Transitoires (TAROT-1) has been founded by the Centre National de la Recherche Scientifique (CNRS), Institut National des Sciences de l'Univers (INSU) and the Carlsberg Fundation. It has been built with the support of the Division Technique of INSU (INSU/DT). We thank the anonymous referee for his/her helpful comments.

\section{References}

Akerlof, C., Balsano, R., Barthelmy, S., et al. 1999, Nature, 398,400

Akerlof, C., Balsano, R., Barthelmy, S., et al. 2000, ApJ, 532, L25

Barthelmy, S. 1997, Proc. of the 4th Huntsville Symp., AIP Conf. Proc., 428, ed. C. A. Meegan, R. D. Preece, \& T. M. Koshut, 99

Bernabei, S., Bartolini, C., di Fabrizio, L., et al. 2000, GCNC, 599,1

Bloom, J. S., Djorgovski, S. G., Gal, R. R., Kulkarni, S. R., \& Kelly, A. 1998a, GCNC, 87, 1

Bloom, J. S., Fail, D. A., Kulkarni, S. R., et al. 1998b, ApJ, 508, L21

Boër, M., Bringer, M., Klotz, A., et al. 1999, A\&AS, 138, 579

Boër, M., \& Gendre, B. 2000, A\&A, 361, L28

Bringer, M., Boër, M., Peignot, C., Fontan, G., \& Merce, C. 2001, Exper. Astrophys., accepted

Castro-Tirado, A. J., Blake, C., Wall, J., Feulner, G. \& Hopp, U. 1999, IAUC, 7332

Costa, E., Frontera, F., Heise, J., et al. 1997, Nature, 387, 783

Costa, E. 1999, A\&AS, 138, 425

Diercks, A. H., Deutsch, E. W., Castander, F. J., et al. 1998, ApJ, 503, L105

Djorgovski, S. G., et al. 1998, IAUC, 6660

Djorgovski, S. G., Kulkarni, S. R., Odewahn, S. C., \& Ebeling, H. 1998, GCNC, 117

Djorgovski, S. G., Goodrich, R., Kulkarni, S. R., et al. 1999, GCNC, 510

Dolan, C., dell'Antonio, I., Jannuzi, B., \& Rhoads, J. 1999, GCNC, 486

Fishman, G., et al. 1989, Proc. Gamma-Ray Observatory science workshop, ed. W. N. Johnson, GSFC Greenbelt, 2
Fruchter, A., Bergeron, L., \& Pian, E. 1997, IAUC, 6674

Fynbo, J. P. U., Jensen, B. L., Hjorth, J., Pedersen, H., \& Gorosabel, J. 2000, GCNC, 576

Gal, R. R., et al. 1998, GCNC, 88

Galama, T. J., Groot, P. J., van Paradijs, J., et al. 1997, IAUC, 6655

Galama, T. J., Briggs, M. S., Wijers, R. A. M., et al. 1999, Nature, 398, 394

Gal-Yam, A., Ofek, E., Maoz, D., \& Leibowitz, E. M. 2000, GCNC, 593

Garcia, L., et al. 1997, IAUC, 6661

Garnavich, P., Jha, S., Stanek, K., Pahre, M., \& Garcia, M. 1999, GCNC, 495

Garnavich, P., Barmby, P., Jha, S., \& Stanek, K. 2000, GCNC, 581

Gehrels, N. 2000, Proc. of the 5th Huntsville Symp., AIP Conf. Proc., 526, ed. R. M. Kippen, S. Mallozzi, \& G. Fishman, 671

Guarnieri, A., Bartolini, C., Masetti, N., et al. 1997, A\&A, 328, 13

Halpern, J. P., \& Fesen, R. 1998, GCNC, 134

Halpern, J. P., et al. 2000, ApJ, in press

Harrison, F. A., Bloom, J. S., Frail, D. A., et al. 1999, ApJ, 523, L121

Henden, A., Luginbuhl, C., Vrba, F., et al. 1999, GCNC, 473

Henden, A. 2000, GCNC, 652

Henden, A., \& Klose, S. 2000, GCNC, 656

Hjorth, J., Andersen, M. I., Pedersen, H., et al. 1998, GCNC, 109

Jensen, B. L., Hjorth, J., Pedersen, H., et al. 1999, GCNC, 454

Jensen, B. L., Pedersen, H., Hjorth, J., et al. 1999, GCNC, 498

Jha, S., Kirshner, R., Stank, K., et al. 1999, GCNC, 476

Kumar, P., \& Panaitescu, A. 2000, ApJ, 541, L51

Kumar, P., \& Piran, T. 2000, ApJ, 535, 152

Kuulkers, E., Antonelli, L. A., Kuiper, L., et al. 2000, ApJ, 538,638

Masetti, N., Palazzi, E., Pian, E., et al. 1999, GCNC, 462

Mészáros, P., \& Rees, M. 1997, ApJ, 476, 232

Metzger, M. 1997, IAUC, 6588

Mignoli, M. 1997, IAUC, 6661

Mirabal, N. 2000, GCNC, 653

Mohan, V., Pandey, A. K., Pandey, S. B., Sagar, R., \& CastroTirado, A. J. 2000, GCNC, 595

Paciesas, W. S., Meegan, C. A., Pendleton, G. N., et al. 1999, ApJS, 122, 465

Panaitescu, A., Mészáros, P., \& Rees, M. 1998, ApJ, 503, 314

Pedersen, H., et al. 1998, ApJ, 503, 314

Pedichini, F., diPaola, A., Stella, L., et al. 1997, A\&A, 327, 36

Pian, E., Amati, L., Antonelli, L. A., et al. 1999, A\&AS, 138, 463

Piran, T. 1999, Phys. Rep., 314, 575

Rees, M., \& Mészáros, P. 1992, MNRAS, 258, 41

Reichart, D. E., Lamb, D. Q., Metzger, M. R., et al. 1999, ApJ, 517,692

Ricker, G. R., HETE Science Team 2001, AAS, 198

Sagar, R., Mohan, V., Pandey, A. K., Pandey, S. B., \& CastroTirado, A. J. 1999, Bull. Astron. Soc. India, 28, 15

Schlegel, D. J., Finkbeiner, D. P., \& Davis, M. 1998, ApJ, 500, 525

Stanek, K. Z., Garnavich, P. M., Kaluzny, J., Pych, W., \& Thompson, I. 1999, ApJ, 522, L39

van Paradijs, J., Groot, P. J., Galama, T., et al. 1997, Nature, 386,686

Veillet, C., \& Boër, M. 2000, GCNC, 598

Vrba, F. J., Henden, A. A., Canzian, B., et al. 2000, ApJ, 528, 254 